\title{
Analysis of the Galaxy Distribution using Multiscale Methods
}

\author{
P. Querre ${ }^{a}$, J.-L. Starck ${ }^{a}$ and V.J. Martínez ${ }^{b}$ \\ ${ }^{a}$ DAPNIA/SEDI-SAP, Service d'Astrophysique, \\ CEA/Saclay, 91191 Gif sur Yvette, France \\ ${ }^{b}$ Observatori Astronòmic, Universitat de València, E-46100 Burjassot, Spain
}

\begin{abstract}
Galaxies are arranged in interconnected walls and filaments forming a cosmic web encompassing huge, nearly empty, regions between the structures. Many statistical methods have been proposed in the past in order to describe the galaxy distribution and discriminate the different cosmological models. We present in this paper preliminary results relative to the use of new statistical tools using the 3D á trous algorithm, the 3D ridgelet transform and the 3D beamlet transform. We show that such multiscale methods produce a new way to measure in a coherent and statistically reliable way the degree of clustering, filamentarity, sheetedness, and voidedness of a dataset.
\end{abstract}

Keywords: Galaxy distribution, large scale structures, wavelet, ridgelet, beamlet, multi-scale methods

\section{INTRODUCTION}

Galaxies are not uniformly distributed throughout the universe. Voids, filaments, clusters, and walls of galaxies can be observed, and their distribution constraints our cosmological theories. Therefore we need statistical methods to compare the catalogs of galaxies to theoretical models and simulations.

The standard approach for testing models is to define a point process which can be characterized by statistical methods. This could be the distribution of galaxies of a specific type in deep surveys or clusters of galaxies. In order to compare models of structure formation, the different distribution of dark matter particles in N-body simulations could be tested as well.

The two-point correlation function $\xi(r)$ has been the primary tool for quantifying large-scale cosmic structure. ${ }^{1}$ Assuming that the galaxy distribution in the Universe is a realization of a stationary and isotropic random process, the two-point correlation function can be defined from the probability $\delta P$ of finding an object within a volume element $\delta V$ at distance $r$ from a randomly chosen object or position inside the volume: $\delta P=n(1+\xi(r)) \delta V$, where $n$ is the mean density of objects. The $\xi(r)$ function measures the clustering properties of objects in a given volume. It is zero for a uniform random distribution, positive (respectively, negative) for a more (respectively, less) clustered distribution. For a hierarchical gravitational clustering or fractal process, $1+\xi(r)$ has a power-law behavior with exponent $D_{2}-3$. Since $\xi(r) \sim r^{-\gamma}$ for the observed galaxy distribution, the correlation dimension for the range where $\xi(r) \gg 1$ is $D_{2} \simeq 3-\gamma$. The two-point correlation function can been generalized to the N-point correlation function. ${ }^{2,3}$ Other statistical measures to characterize the spatial distribution of galaxies have also been developed, ${ }^{4}$ such as multifractals, ${ }^{5}$ the Minkowski functionals ${ }^{6,7}$ or the $J$ function $^{8,9}$ which is defined as the ratio $J(r)=\frac{1-G(r)}{1-F(r)}$, where $F$ is the distribution function of the distance $r$ of an arbitrary point in $\mathbf{R}^{3}$ to the nearest object, and $G$ is the distribution function of the distance $r$ of an object to the nearest object. Wavelets have also been used for analyzing the projected 2D or the 3D galaxy distribution. ${ }^{10-14}$

Other multiscale methods have recently emerged, the beamlet transform ${ }^{15,16}$ and the ridgelet transform, ${ }^{17}$ which allows us to better represent data containing respectively filaments and sheets, while wavelets represent

Further author information: Send correspondence to jstarck@cea.fr 
well isotropic features (i.e. cluster in 3D). As each of these three transforms represents perfectly one kind of feature, all of them are useful and should be used to describe a given catalog.

Section 2, 3 and 4 describes respectively the 3D wavelet transform, the 3D ridgelet transform and the 3D beamlet transform, and numerical experiments are given in section 4.

\section{THE 3D WAVELET TRANSFORM}

\section{The à trous Isotropic Wavelet Transform}

The 'a trous algoritm ${ }^{18}$ of a cube $c$ produces, at each scale $j$, a set of zero-mean coefficient values $\left\{w_{j}\right\}$. This set $\left\{w_{j}\right\}$ has the same number of pixels as the input cube (this wavelet transform is redundant). Using a wavelet $\psi$ defined as the difference between the scaling functions of two successive scales,

$$
\frac{1}{2} \psi\left(\frac{x}{2}, \frac{y}{2}, \frac{z}{2}\right)=\phi(x, y, z)-\phi\left(\frac{x}{2}, \frac{y}{2}, \frac{z}{2}\right),
$$

the original cube $c=c_{0}$, can be expressed as the sum of all the wavelet scales $\left\{w_{j}\right\}$ and the last smoothed array $c_{J}$

$$
c_{0, x, y, z}=c_{J, x, y, z}+\sum_{j=1}^{J} w_{j, x, y, z},
$$

The set $w=\left\{w_{1}, w_{2}, \ldots, w_{J}, c_{J}\right\}$ represents the wavelet transform of the data. if we denote $\mathcal{W}$ the wavelet transform operator and $N$ the pixel number of $c$, the wavelet transform $w(w=\mathcal{W} c)$ has $(J+1) N$ pixels (redundancy factor of $J+1$ ). The scaling function $\phi$ is generally chosen as a spline of degree 3 and the 3D implementation is based on three 1D sets of (separable) convolutions. Like the scaling function $\phi$, the wavelet function $\psi$ is isotropic (point symmetric). More details can be found in. ${ }^{18,19}$

For each $a>0, b_{1}, b_{2}, b_{3} \in \mathbf{R}^{3}$, the wavelet is defined by

$$
\begin{aligned}
\psi_{a, b_{1}, b_{2}, b_{3}}: & \mathbf{R}^{3} \rightarrow \mathbf{R} \\
& \psi_{a, b_{1}, b_{2}, b_{3}}\left(x_{1}, x_{2}, x_{3}\right)=a^{-1 / 2} \cdot \psi\left(\frac{x_{1}-b_{1}}{a}, \frac{x_{2}-b_{2}}{a}, \frac{x_{3}-b_{3}}{a}\right)
\end{aligned}
$$

Given a function $f \in L^{2}\left(\mathbf{R}^{3}\right)$, we define its wavelet coefficients by:

$$
\begin{array}{ll}
\mathcal{W}_{f}: & \mathbf{R}^{4} \rightarrow \mathbf{R} \\
& \mathcal{W}_{f}\left(a, b_{1}, b_{2}, b_{3}\right)=\int \bar{\psi}_{a, b_{1}, b_{2}, b_{3}}(\mathbf{x}) f(\mathbf{x}) d \mathbf{x} .
\end{array}
$$

Figure 1 shows an example of 3D wavelet function.

\section{THE 3D RIDGELET TRANSFORM}

The two-dimensional continuous ridgelet transform of a function $f \in L^{2}\left(\mathbf{R}^{2}\right)$ is defined as follows. ${ }^{17}$ We first select a smooth function $\psi \in L^{2}(\mathbf{R})$, we assume that $\psi$ satisfies the admissibility condition

$$
\int|\hat{\psi}(\xi)|^{2} /|\xi| d \xi<\infty
$$

which holds if $\psi$ has a sufficient decay and a vanishing mean $\int \psi(t) d t=0$ ( $\psi$ can be normalized so that it has unit energy $\left.1 /(2 \pi) \int|\hat{\psi}(\xi)|^{2} d \xi=1\right)$.

For each $a>0, b \in \mathbf{R}$ and $\theta_{1} \in[0,2 \pi[$, we define the ridgelet by

$$
\begin{aligned}
\psi_{a, b, \theta_{1}}: & \mathbf{R}^{2} \rightarrow \mathbf{R} \\
& \psi_{a, b, \theta_{1}}\left(x_{1}, x_{2}\right)=a^{-1 / 2} \cdot \psi\left(\left(x_{1} \cos \theta_{1}+x_{2} \sin \theta_{1}-b\right) / a\right) ;
\end{aligned}
$$

Given a function $f \in L^{2}\left(\mathbf{R}^{2}\right)$, we define its ridgelet coefficients by:

$$
\begin{aligned}
\mathcal{R}_{f}: & \mathbf{R}^{3} \rightarrow \mathbf{R} \\
& \mathcal{R}_{f}\left(a, b, \theta_{1}\right)=\int \bar{\psi}_{a, b, \theta_{1}}(\mathbf{x}) f(\mathbf{x}) d \mathbf{x} .
\end{aligned}
$$



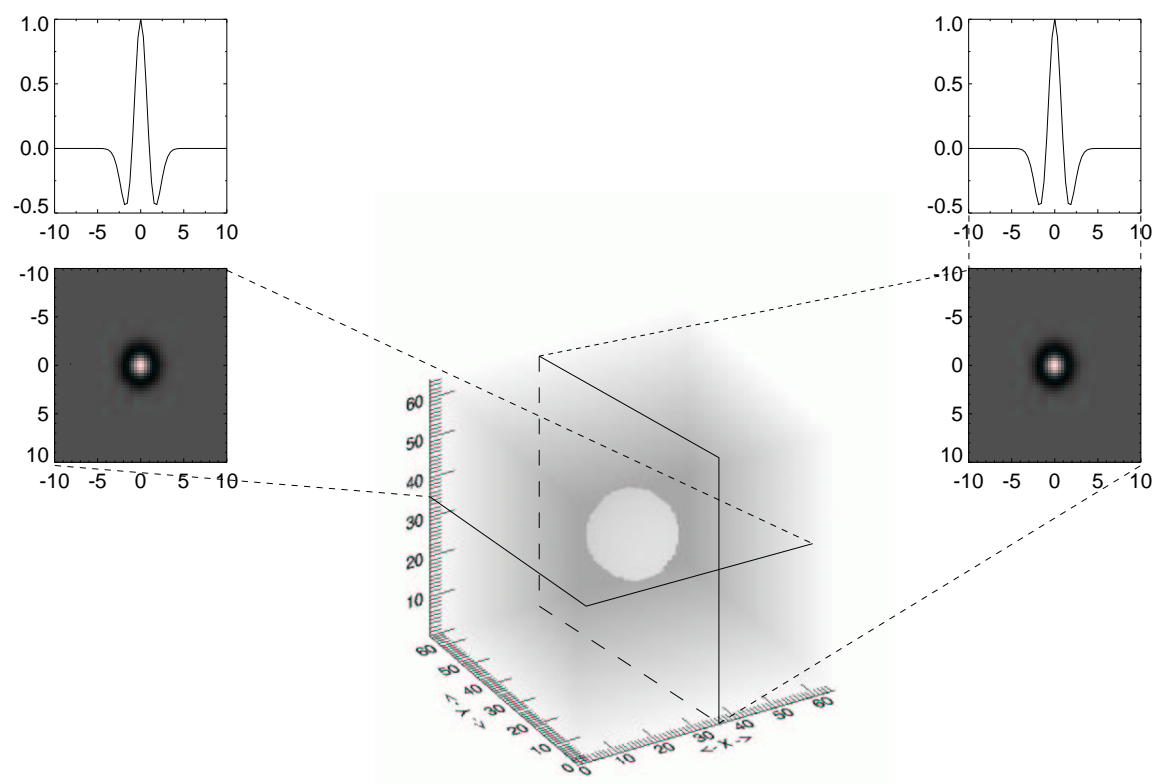

Figure 1. Example of wavelet function.

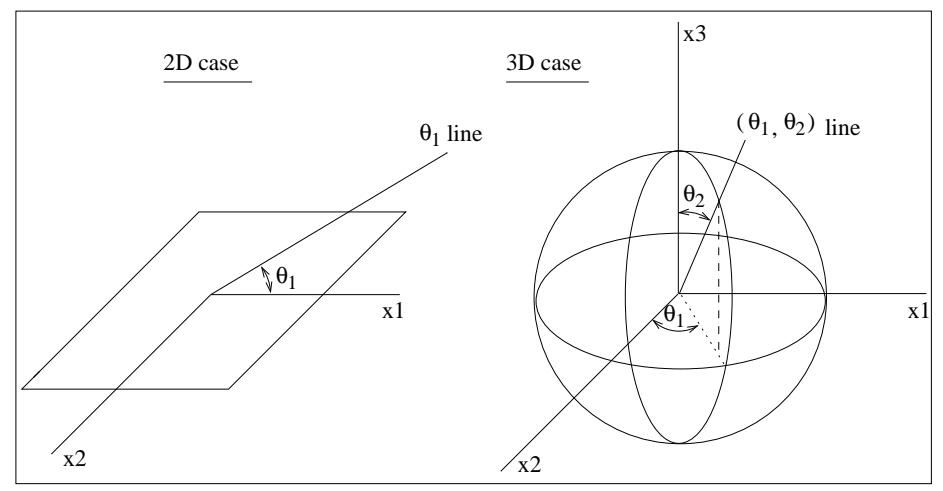

Figure 2. Definition of angle $1 \theta_{1}$ and $\theta_{2}$ in $\mathbf{R}^{2}$ and $\mathbf{R}^{3}$

It has been shown ${ }^{17}$ that the ridgelet transform is precisely the application of a 1-dimensional wavelet transform to the slices of the Radon transform (where the angular variable $\theta_{1}$ is constant). This method is therefore optimal to detect lines of the size of the image (the integration increase as the length of the line). More details on the implementation of the digital ridgelet transform can be found in. ${ }^{20}$

Figure 3 (left) shows an example ridgelet function. This function is constant along lines $x_{1} \cos \theta+x_{2} \sin \theta=$ const. Transverse to these ridges it is a wavelet (see figure 3 (right).

\section{From 2D to 3D}

The three-dimensional continuous ridgelet transform of a function $f \in L^{2}\left(\mathbf{R}^{3}\right)$ is given by:

$$
\begin{aligned}
\mathcal{R}_{f}: & \mathbf{R}^{4} \rightarrow \mathbf{R} \\
& \mathcal{R}_{f}\left(a, b, \theta_{1}, \theta_{2}\right)=\int \bar{\psi}_{a, b, \theta_{1}, \theta_{2}}(\mathbf{x}) f(\mathbf{x}) d \mathbf{x} .
\end{aligned}
$$

where $a>0, b \in \mathbf{R}, \theta_{1} \in\left[0,2 \pi\left[\right.\right.$ and $\theta_{2} \in[0, \pi[$. 

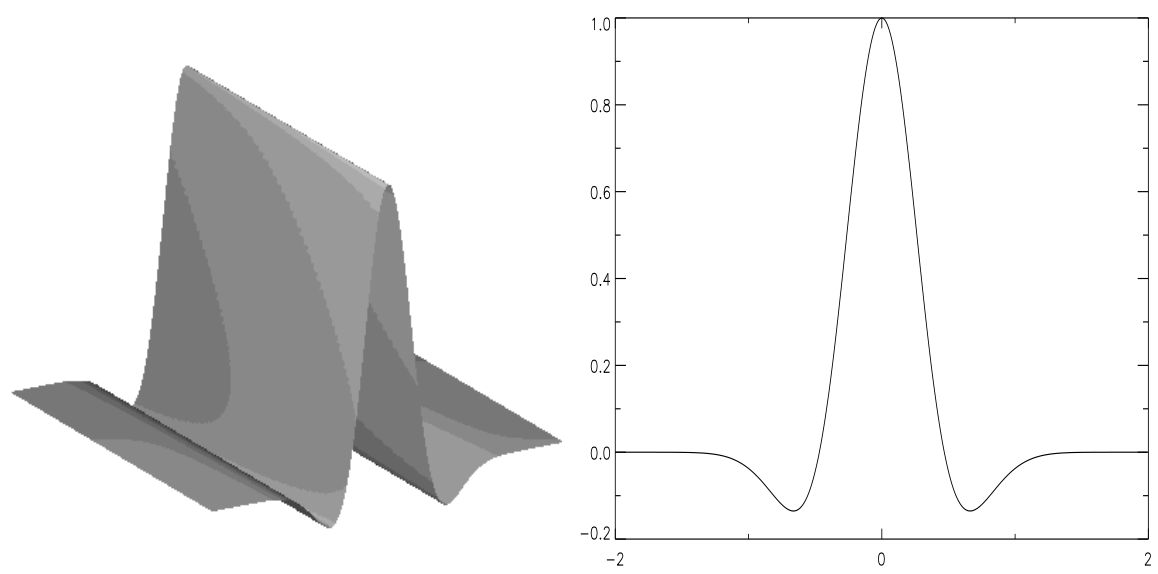

Figure 3. Example of $2 \mathrm{D}$ ridgelet function.

The ridgelet function is defined by:

$$
\begin{aligned}
\psi_{a, b, \theta_{1}, \theta_{2}}: & \mathbf{R}^{3} \rightarrow \mathbf{R} \\
& \psi_{a, b, \theta_{1}, \theta_{2}}\left(x_{1}, x_{2}, x_{3}\right)=a^{-1 / 2} \cdot \psi\left(\left(x_{1} \cos \theta_{1} \cos \theta_{2}+x_{2} \sin \theta_{1} \cos \theta_{2}+x_{3} \sin \theta_{2}-b\right) / a\right) ;
\end{aligned}
$$

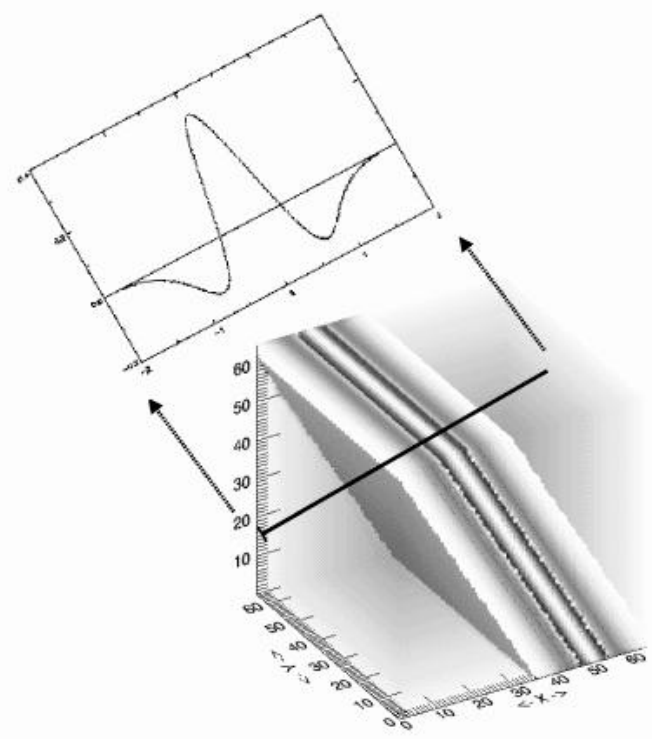

Figure 4. Example of ridgelet function.

Figure 4 shows an example of ridgelet function. It is a wavelet function in the direction defined by the line $\left(\theta_{1}\right.$, thet $\left._{2}\right)$, and it is constant along the orthogonal plane to this line.

As in the $2 \mathrm{D}$ case, the $3 \mathrm{D}$ ridgelet transform can be built by extracting lines in the Fourier domain. Let $c\left(i_{1}, i_{2}, i_{3}\right)$ a cube of size $(N, N, N)$, the algorithm consists in the following steps:

1. $3 D$-FFT. Compute $\hat{c}\left(k_{1}, k_{2}, k_{3}\right)$, the three-dimensional FFT of the cube $c\left(i_{1}, i_{2}, i_{3}\right)$. 


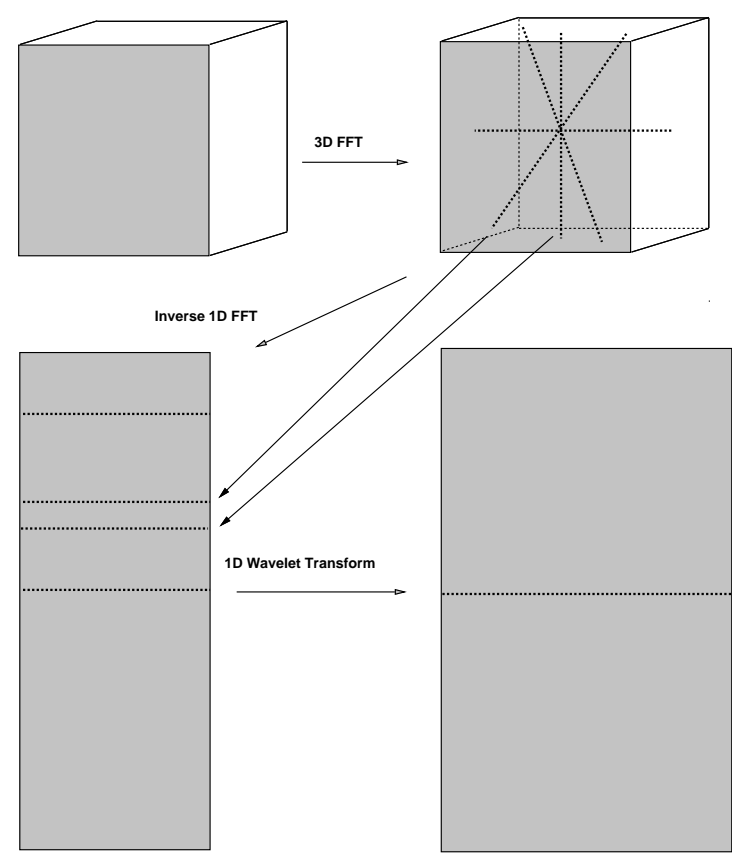

Figure 5. 3D ridgelet transform flowgraph.

2. Cartesian to Spherical Conversion. Using an interpolation scheme, substitute the sampled values of $\hat{c}$ obtained on the cartesian coordinate system $\left(k_{1}, k_{2}, k_{3}\right)$ with sampled values of on a spherical coordinate system $\left(\theta_{1}, \theta_{2}, \rho\right)$.

3. Extract lines. Extract the $3 N^{2}$ lines (size $N$ ) passing throught the origin and the boundary of $\hat{c}$.

4. 1D-IFFT. Compute the one-dimensional inverse FFT on each line.

5. 1D-WT. Compute the one-dimensional wavelet transform on each line.

Figure 5 the 3D ridgelet transform flowgraph. The 3D ridgelet transform allows us to detect sheets in a cube.

\section{Local 3D Ridgelet Transform}

The ridgelet transform is optimal to find sheets of the size of the cube. To detect smaller sheets, a partitioning must be introduced. ${ }^{21}$ The cube $c$ is decomposed into blocks of lower side-length $b$ so that for a $N * N * N$ cube, we count $N / b$ blocks in each direction. After the block partitioning, The detection is therefore optimal for sheets of size $b \times b$ and of thickness $a_{j}, a_{j}$ corresponding to the different dyadic scales used in the transformation.

\section{THE 3D BEAMLET TRANSFORM}

Let $\psi \in L^{2}\left(\mathbf{R}^{2}\right)$ a smooth function satisfying the admissibility condition (a 2D wavelet function), the threedimensional continuous beamlet transform of a function $f \in L^{2}\left(\mathbf{R}^{3}\right)$ is given by:

$$
\begin{aligned}
\mathcal{B}_{f}: & \mathbf{R}^{5} \rightarrow \mathbf{R} \\
& \mathcal{B}_{f}\left(a, b_{1}, b_{2}, \theta_{1}, \theta_{2}\right)=\int \bar{\psi}_{a, b, \theta_{1}, \theta_{2}}(\mathbf{x}) f(\mathbf{x}) d \mathbf{x} .
\end{aligned}
$$

where $a>0, b_{1}, b_{2} \in \mathbf{R}, \theta_{1} \in\left[0,2 \pi\left[\right.\right.$ and $\theta_{2} \in[0, \pi[$.

The beamlet function is defined by:

$$
\begin{aligned}
& \psi_{a, b_{1}, b_{2}, \theta_{1}, \theta_{2}}: \quad \mathbf{R}^{3} \rightarrow \mathbf{R} \\
& \psi_{a, b_{1}, b_{2}, \theta_{1}, \theta_{2}}\left(x_{1}, x_{2}, x_{3}\right)=a^{-1 / 2} \cdot \psi\left(\quad\left(-x_{1} \sin \theta_{1}+x_{2} \cos \theta_{1}+b_{1}\right) / a,\right. \\
& \left.\left(x_{1} \cos \theta_{1} \cos \theta_{2}+x_{2} \sin \theta_{1} \cos \theta_{2}-x_{3} \sin \theta_{2}+b_{2}\right) / a\right) \text {; }
\end{aligned}
$$




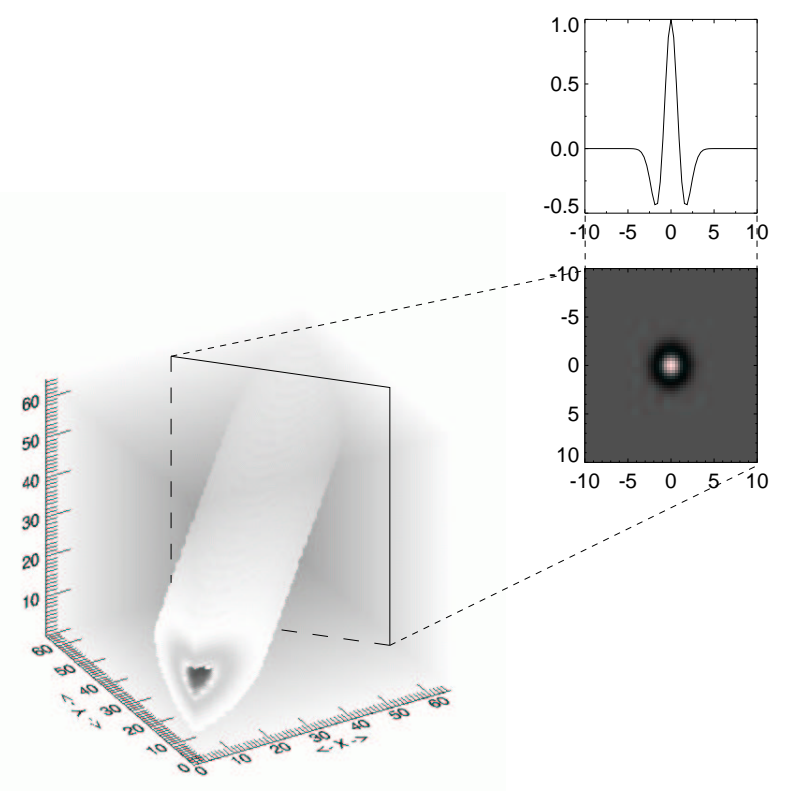

Figure 6. Example of beamlet function.

Figure 4 shows an example of beamlet function. It is constant along lines of direction $\left(\theta_{1}, \theta_{2}\right)$, and a $2 \mathrm{D}$ wavelet function along plane orthogonal to this direction.

The 3D beamlet transform can be built using the "Generalized projection-slice theorem". ${ }^{22}$ Let

- $f(\mathbf{x})$ an $n$ dimensional function,

- $\mathcal{R}_{a d_{m}} f$ its $\mathrm{m}$-dimensional partial radon transform along the first $\mathrm{m}$ cardinal directions, $m<n, \mathcal{R} a d_{m} f$ is a function of $\left(p, \mu_{m} ; x_{m+1}, \ldots, x_{n}\right), \boldsymbol{\mu}_{m}$ a unit directional vector in $\mathcal{R} a d_{m}$ (note that for a given projection angle, the $m$ dimensional partial radon transform of $f(\mathbf{x})$ has $(n-m)$ untransformated spatial dimension and a $(n-m+1)$ dimensional projection profile),

- $\{\mathcal{F} f\}(\mathbf{k})$ its Fourier transform $(\mathbf{x}$ and $\mathbf{k}$ are conjugate variable pairs of $\mathcal{F})$,

The Fourier transform of the $m$ dimensional partial radom transform $\mathcal{R}_{m} f$ is related to the Fourier transform of $f(\mathcal{F} f)$ by the following relation

$$
\left\{\mathcal{F}_{n-m+1} \mathcal{R a d}_{m} f\right\}\left(k, k_{m+1}, \ldots, k_{n}\right)=\{\mathcal{F} f\}\left(k \boldsymbol{\mu}_{m}, k_{m+1}, \ldots, k_{n}\right)
$$

Let $c\left(i_{1}, i_{2}, i_{3}\right)$ a cube of size $(N, N, N)$, the Beamlet algorithm consists in the following steps:

1. 3D-FFT. Compute $\hat{c}\left(k_{1}, k_{2}, k_{3}\right)$, the three-dimensional FFT of the cube $c\left(i_{1}, i_{2}, i_{3}\right)$.

2. Cartesian to Spherical Conversion. Using an interpolation scheme, substitute the sampled values of $\hat{c}$ obtained on the cartesian coordinate system $\left(k_{1}, k_{2}, k_{3}\right)$ with sampled values of on a spherical coordinate system $\left(\theta_{1}, \theta_{2}, \rho\right)$.

3. Extract planes. Extract the $3 N^{2}$ planes (of size $N \times N$ ) passing throught the origin (each line used in the $3 \mathrm{D}$ ridgelet transform defines set of orthogonal planes, we take the one which include the origin). 


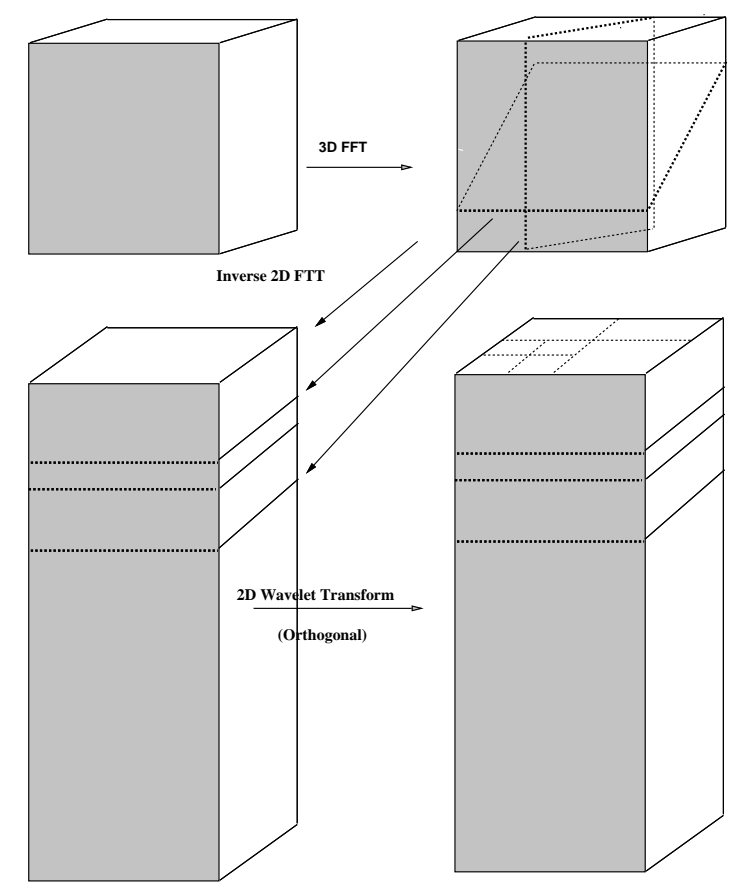

Figure 7. 3D beamlet transform flowgraph.

4. 2D-IFFT. Compute the two-dimensional inverse FFT on each plane.

5. 2D-WT. Compute the two-dimensional wavelet transform on each plane.

Figure 7 the 3D beamlet transform flowgraph. The 3D beamlet transform allows us to detect filament in a cube. The beamlet transform algorithm presented in this section differs from the one presented in, ${ }^{23}$ and relation between both algorithms is given in. ${ }^{16}$

\section{EXPERIMENTS}

\subsection{Experiment 1}

We have simulated three data set containing respectively a cluster, a plane and a line. On each data set, Poisson noise have been added with eight different background levels. Then we have applied the three transforms on the 24 simulated data set. The coefficients distribution related to each transformation is normalized using twenty realizations of a 3D flat distribution with a Poisson noise and which have the same number of counts as in the data.

Figure 8 shows, from top to bottom, the maximum value of the normalized distribution versus the noise level for our three simulated data set. As expected, wavelets, ridgelets and beamlets are respectively the best for clusters, sheets and lines detection. It must also be underlined that a feature can be detected with a very high signal-to-noise ratio in a given basis, and and not detected in another basis. For example, the wall is detected at more than $60 \sigma$ by the ridgelet transform, and less than $5 \sigma$ by the wavelet transform. The line is detected almost at $10 \sigma$ by the beamlet transform, and is under a $3 \sigma$ detection level using wavelets. These results shows the importance of using several transforms for an optimal detection of all features contained in a data set.

\subsection{Experiment 2}

We use here two simulated data sets to illustrate the discriminative power of the multiscale methods. The first one is a simulation from stochastic geometry. It is based on a Voronoi model. The second one is a mock 

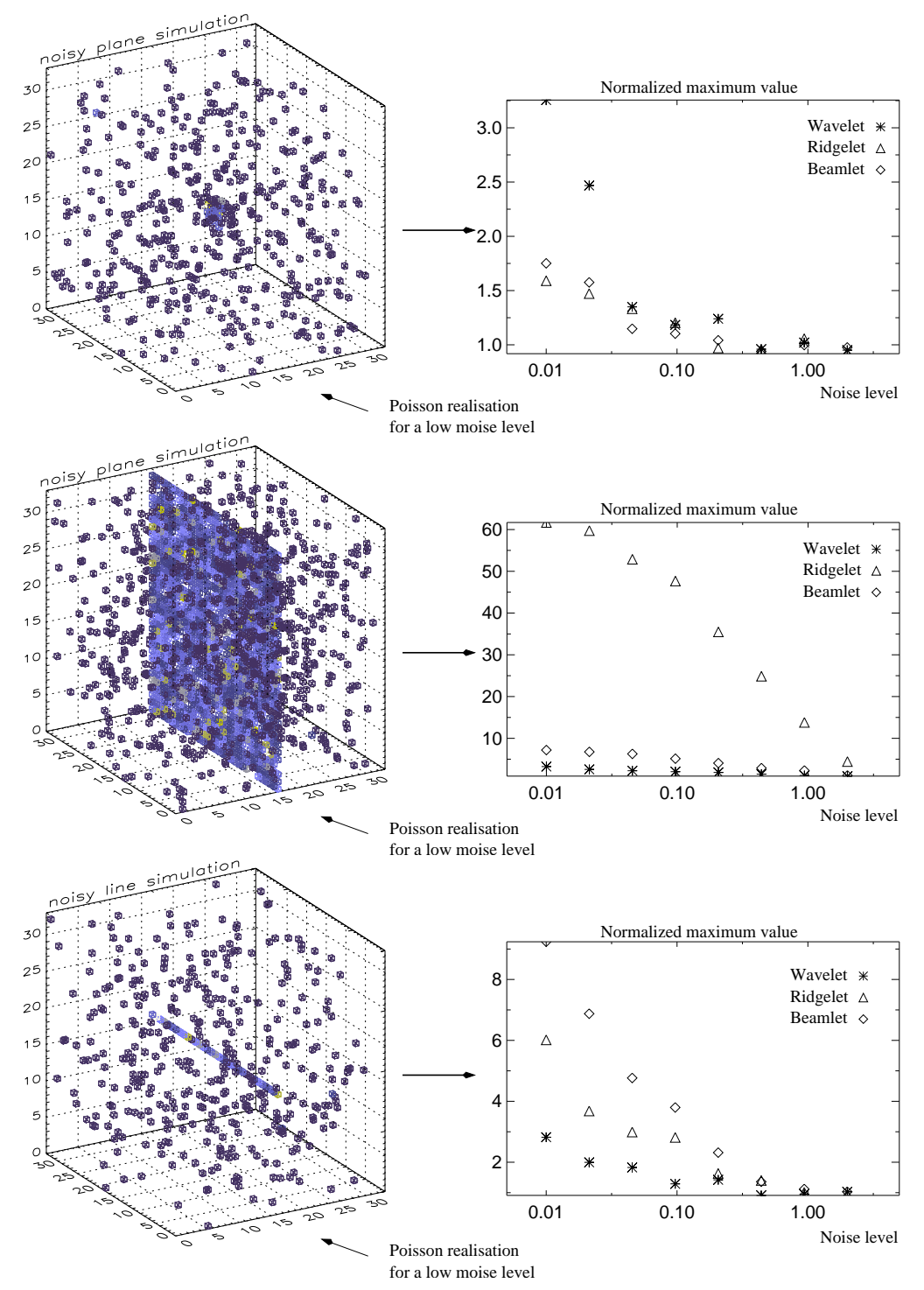

Figure 8. Simulation of cubes containing a cluster (top), a plane (middle) and a line (bottom).

catalog of the galaxy distribution drawn from a $\Lambda$-CDM N-body cosmological model. ${ }^{24}$ Both processes have very similar two-point correlation functions at small scales, although they look quite different and have been generated following completely different algorithms.

- the first comes from a Voronoi simulation: We locate a point in each of the vertices of a Voronoi tessellation of 1.500 cells defined by 1500 nuclei distributed following a binomial process. There are 10085 vertices lying within a box of $141.4 h^{-1} \mathrm{Mpc}$ side.

- the second point pattern represents the galaxy positions extracted from a cosmological $\Lambda$-CDM N-body simulation. The simulation has been carried out by the Virgo consortium and related groups (see http://arXiv/org/abs/astro-ph/0007362). The simulation is a low-density $(\Omega=0.3)$ model with cosmological constant $\Lambda=0.7$. It is, therefore, the closer set to the real galaxy distribution. ${ }^{24}$ There are 15445 galaxies within a box with side $141.3 h^{-1} \mathrm{Mpc}$. Galaxies in this catalog have stellar masses exceeding $2 \times 10^{10} M_{\odot}$. 

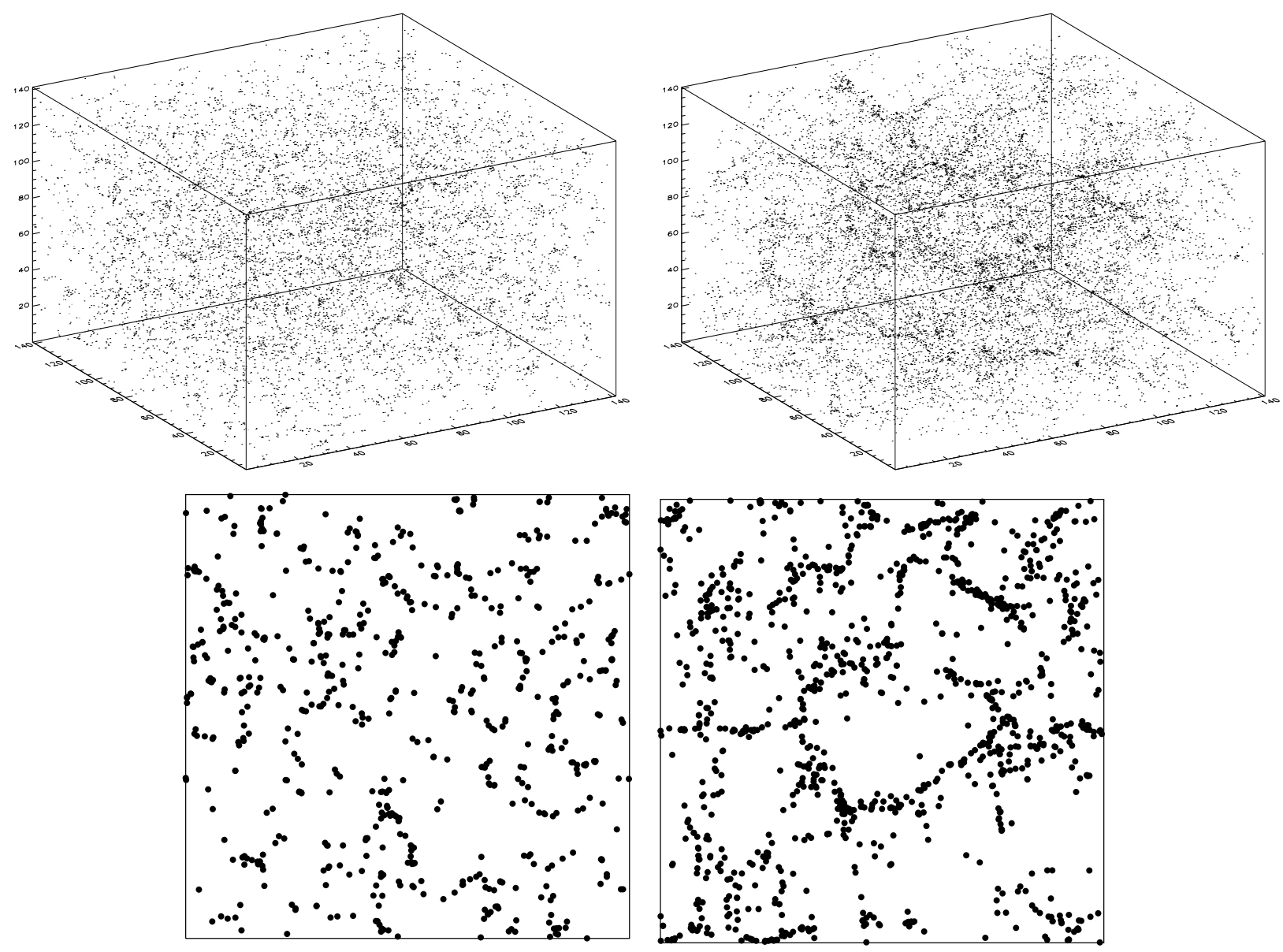

Figure 9. Simulated data sets. Top, the Voronoi vertices point pattern (left) and the galaxies of the GIF $\Lambda$-CDM N-body simulation (right). The bottom panels show one $10 h^{-1}$ width slice of the each data set.

Figure 9 shows the two simulated data set, and Figure 10 shows the two-point correlation function curve for the two point processes. The two point fields are different, but as it can be seen in Fig. 10, both have very similar two-point correlation functions in a huge range of scales (2 decades).

We have applied the three transforms to each data set, and we have calculated the skewness vector $S=$ $\left(s_{w}^{j}, s_{r}^{j}, s_{b}^{j}\right)$ and the kurtosis vector $K=\left(k_{w}^{j}, k_{r}^{j}, k_{b}^{j}\right)$ at each scale $j . s_{w}^{j}, s_{r}^{j}, s_{b}^{j}$ are respectively the skewness at scale $j$ of the wavelet coefficients, the ridgelet coefficients and the beamlet coefficients. $k_{w}^{j}, k_{r}^{j}, k_{b}^{j}$ are respectively the kurtosis at scale $j$ of the wavelet coefficients, the ridgelet coefficients and the beamlet coefficients. Figure 11 shows the kurtosis and the skewness vectors of the two data set at the two first scales. On the contrary to the two-point correlation function, this analysis shows strong differences between the two data set, particularly on the wavelet axis, which indicates that the second data contains more, or with a higher density, clusters than the first one.

\section{ACKNOWLEDGMENTS}

We thank the Virgo consortium and the GIF project for providing us with the $\Lambda$-CDM simulation. VJM thanks E. Saar, R. van de Weygaert, S. Paredes, M.J. Pons-Bordería, and B. López-Martí for kindly allowing us to use some of their data and results. VJM acknowledge support of the Spanish MCyT project AYA2000-2045. 


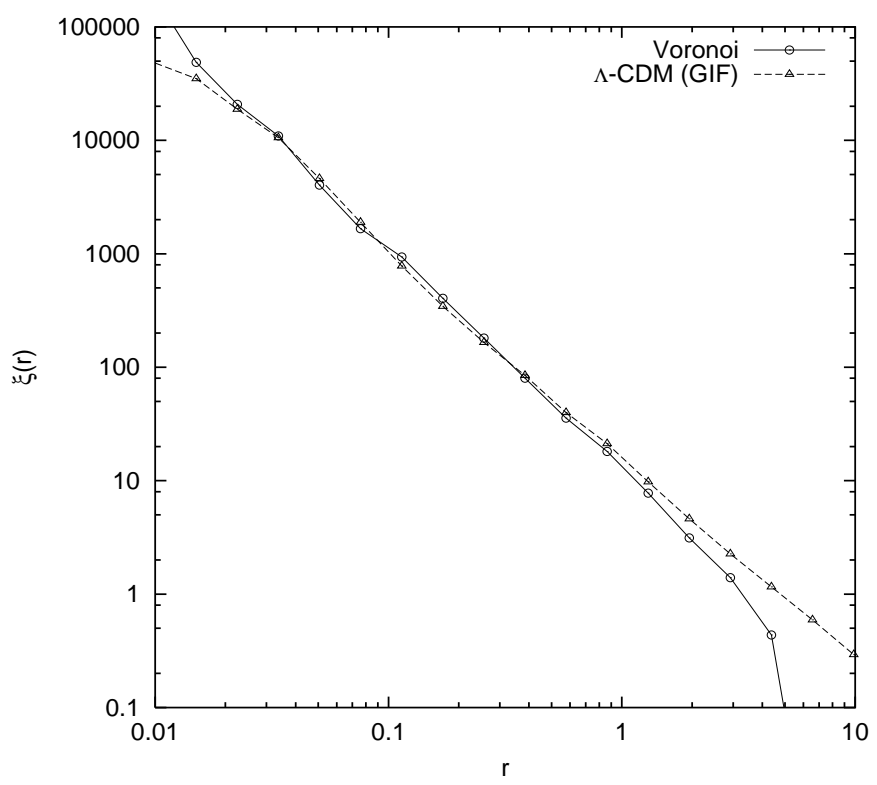

Figure 10. The two-point correlation function of the Voronoi vertices process and the GIF $\Lambda$-CDM N-body simulation. They are very similar in the range $[0.02,2] h^{-1} \mathrm{Mpc}$.

\section{REFERENCES}

1. P. Peebles, The Large-Scale Structure of the Universe, Princeton University Press, 1980.

2. S. Szapudi and A. S. Szalay, "A new class of estimators for the N-point correlations," Astrophysical Journal Letters 494, p. L41, Feb. 1998.

3. P. Peebles, "The galaxy and mass N-point correlation functions: a blast from the past," in Historical Development of Modern Cosmology, V. Martínez, V. Trimble, and M. Pons-Bordería, eds., ASP Conference Series, (Astronomical Society of the Pacific), 2001.

4. V. J. Martínez and E. Saar, Statistics of the Galaxy Distribution, Chapman and Hall/CRC press, Boca Raton, 2002.

5. V. J. Martínez, B. J. T. Jones, R. Domínguez-Tenreiro, and R. van de Weygaert, "Clustering paradigms and multifractal measures," Astrophysical Journal 357, pp. 50-61, 1990.

6. K. R. Mecke, T. Buchert, and H. Wagner, "Robust morphological measures for large-scale structure in the Universe," Astronomy and Astrophysics 288, pp. 697-704, Aug. 1994.

7. M. Kerscher, "Statistical analysis of large-scale structure in the Universe," in Statistical Physics and Spatial Satistics: The Art of Analyzing and Modeling Spatial Structures and Pattern Formation, K. Mecke and D. Stoyan, eds., Lecture Notes in Physics 554, 2000.

8. M. V. Lieshout and A. Baddeley, "A non parametric measure of spatial interaction in point patterns," Statistica Neerlandica 50, pp. 344-361, 1996.

9. M. Kerscher, M. J. Pons-Bordería, J. Schmalzing, R. Trasarti-Battistoni, T. Buchert, V. J. Martínez, and R. Valdarnini, "A global descriptor of spatial pattern interaction in the galaxy distribution," Astrophysical Journal 513, pp. 543-548, 1999.

10. E. Escalera, E. Slezak, and A. Mazure, "New evidence for subclustering in the Coma cluster using the wavelet analysis," Astronomy and Astrophysics 264, pp. 379-384, Oct. 1992.

11. E. Slezak, V. de Lapparent, and A. Bijaoui, "Objective detection of voids and high density structures in the first CfA redshift survey slice," Astrophysical Journal 409, pp. 517-529, 1993.

12. V. J. Martínez, S. Paredes, and E. Saar, "Wavelet analysis of the multifractal character of the galaxy distribution," Monthly Notices of the Royal Astronomical Society 260, pp. 365-375, 1993. 
Skewness, scale 1

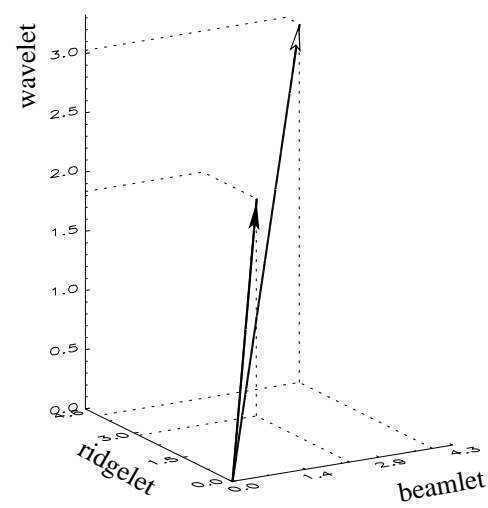

Kurtosis, scale 1

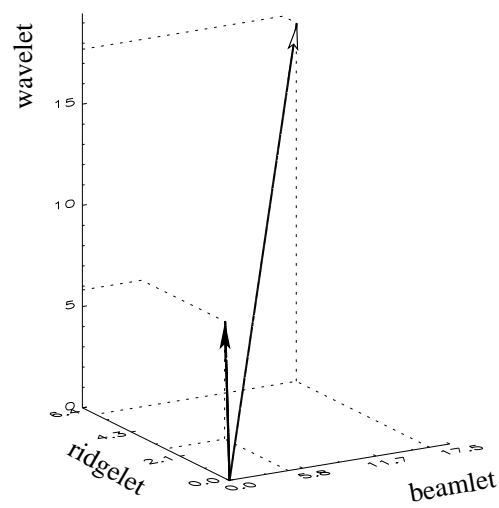

Skewness, scale 2

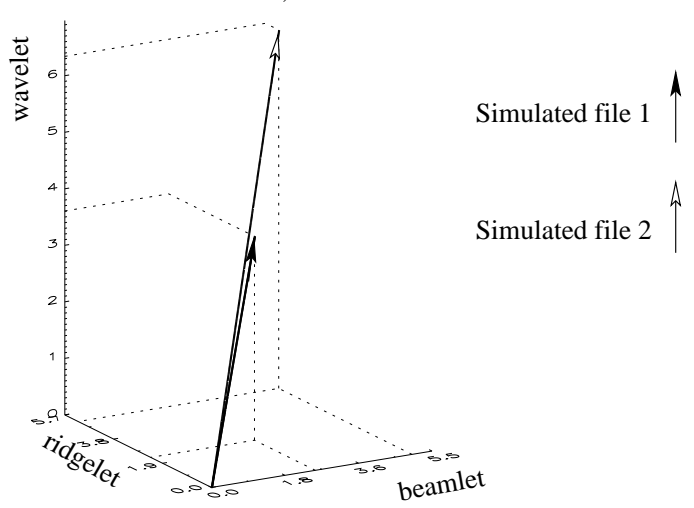

Kurtosis, scale 2

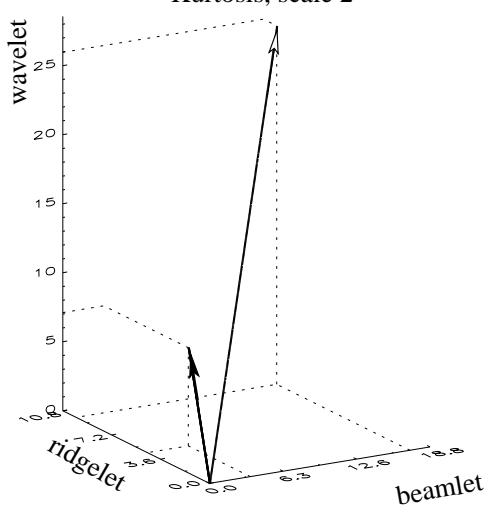

Figure 11. Skewness and kurtosis for the two simulated data set.

13. A. Pagliaro, V. Antonuccio-Delogu, U. Becciani, and M. Gambera, "Substructure recovery by threedimensional discrete wavelet transforms," Monthly Notices of the Royal Astronomical Society 310, pp. 835841, Dec. 1999.

14. T. Kurokawa, M. Morikawa, and H. Mouri, "Scaling analysis of galaxy distribution in the Las Campanas Redshift Survey data," Astronomy and Astrophysics 370, pp. 358-364, May 2001.

15. D. Donoho and X. Huo, Lecture Notes in Computational Science and Engineering, ch. Beamlets and Multiscale Image Analysis. Springer, 2001.

16. D. Donoho and O. Levi, "Fast X-Ray and Beamlet Transforms for Three-Dimensional Data," tech. rep., Statistics, Stanford University, 1999.

17. E. Candès and D. Donoho, "Ridgelets: the key to high dimensional intermittency?," Philosophical Transactions of the Royal Society of London A 357, pp. 2495-2509, 1999.

18. J.-L. Starck, F. Murtagh, and A. Bijaoui, Image Processing and Data Analysis: The Multiscale Approach, Cambridge University Press, 1998.

19. J.-L. Starck and F. Murtagh, Astronomical Image and Data Analysis, Springer-Verlag, 2002. in press.

20. J.-L. Starck, E. Candès, and D. Donoho, "The curvelet transform for image denoising," IEEE Transactions on Image Processing 11(6), pp. 131-141, 2002.

21. E. J. Candès, "Harmonic analysis of neural netwoks," Applied and Computational Harmonic Analysis 6 , pp. 197-218, 1999.

22. P. C. L. Zhi-Oei Liang, Principle of Magnetic Resonance Imaging, IEEE Press, 2000.

23. D. Donoho, O. Levi, J.-L. Starck, and V. M. nez, "Multiscale geometric analysis for 3-d catalogues," in SPIE conference on Astronomical Telescopes and Instrumentation: Astronomical Data Analysis II, Waikoloa, Hawaii, 22-28 August, J.-L. Starck and F. Murtagh, eds., 4847, SPIE, 2002. 
24. G. Kauffmann, J. M. Colberg, A. Diaferio, and S. D. M. White, "Clustering of galaxies in a hierarchical universe - I. Methods and results at $\mathrm{z}=0$," Monthly Notices of the Royal Astronomical Society 303, pp. 188206, 1999. 